\title{
Differentiating Pediatric Rhabdomyosarcoma and Langerhans Cell Histiocytosis of the Temporal Bone by Imaging Appearance
}

\author{
(1) K.M. Chevallier, ${ }^{\circledR}$ R.H. Wiggins, ${ }^{\circ}$ N.A. Quinn, and ${ }^{\circledR}$ R.K. Gurgel
}

\begin{abstract}
BACKGROUND AND PURPOSE: Rhabdomyosarcoma and Langerhans cell histiocytosis are malignant lesions that can affect the skull base with similar radiographic characteristics on CT and MR imaging. We hypothesized that location within the temporal bone determined radiographically can provide useful adjunctive information in differentiating these distinct neoplasms.
\end{abstract}

MATERIALS AND METHODS: We identified patients with Langerhans cell histiocytosis and rhabdomyosarcoma by using an imaging data base and International Classification of Diseases, Ninth Revision codes at a tertiary care academic medical center. Cross-sectional images were reviewed by a neurotologist and neuroradiologist, who evaluated the location of the lesions and scored each subsite-middle ear, mastoid, petrous apex, retrosigmoid/posterior fossa—on a scale of 0 (no involvement), 1 (partial), or 2 (complete involvement).

RESULTS: We identified 12 patients representing 14 cases of Langerhans cell histiocytosis, and 9 patients representing 9 cases of rhabdomyosarcoma. For patients with Langerhans cell histiocytosis, mastoid involvement was rated 23/28 (82\%) compared with 6/18 (33\%) with rhabdomyosarcoma $(P=.001)$. Langerhans cell histiocytosis was present in only the anterior portion of the temporal bone (petrous apex and middle ear) in 1 case $(7.1 \%)$ and in the anterior portion of the temporal bone only in $5 / 9(55 \%)$ cases of rhabdomyosarcoma $(P=.018)$. The cortical bone was more commonly involved in Langerhans cell histiocytosis, $11 / 28(39 \%)$ of cases compared with $2 / 18$ (11\%) cases in rhabdomyosarcoma $(P<.05)$.

CONCLUSIONS: These results indicate that lesions involving only the anterior portion of the temporal bone (petrous apex and middle ear) are more likely to be rhabdomyosarcoma. Lesions involving the mastoid are more likely to be Langerhans cell histiocytosis. This difference in primary location may be helpful in predicting the pathology of these lesions on the basis of imaging.

ABBREVIATIONS: $L C H=$ Langerhans cell histiocytosis; RMS = rhabdomyosarcoma

$\mathbf{R}^{\mathrm{h}}$ habdomyosarcoma (RMS) and Langerhans cell histiocytosis $(\mathrm{LCH})$ are both rare but potentially fatal neoplasms of the pediatric population. These diseases are distinct pathologically: RMS is a soft-tissue sarcoma arising from mesenchymal cells of the middle ear or skeletal muscle, ${ }^{1}$ while $\mathrm{LCH}$ tumors arise from the abnormal proliferation of Langerhans cells (ie, dendritic cells). ${ }^{2}$

Both lesions are commonly found in the head and neck region,

Received August 21, 2015; accepted after revision November 30.

From the Division of Otolaryngology, Head and Neck Surgery (K.M.C. R.H.W., N.Q., R.K.G.), University of Utah, Salt Lake City, Utah; and Department of Radiology (R.H.W.), University of Utah Hospital, Salt Lake City, Utah.

Paper previously presented at: American Society for Pediatric Otolaryngology Poster Session at the Combined Otolaryngology Spring Meeting, May 14-18, 2014; Las Vegas, Nevada.

Please address correspondence to Keely Chevallier, MD, Division of Otolaryngology, 50 N Medical Dr, 3C120, Salt Lake City, UT 84132; e-mail: keely.chevallier@hsc. utah.edu

http://dx.doi.org/10.3174/ajnr.A4676
$30 \%-70 \%$ in $\mathrm{RMS}^{3}$ and $60 \%$ in $\mathrm{LCH} .{ }^{4}$ Less frequently, the temporal bone is involved, with only $8 \%-10 \%$ of cases of RMS of the head and neck ${ }^{3}$ and $19 \%-25 \%$ of LCH cases in this location. ${ }^{4}$ The initial presentation for both diseases is variable and can range from a palpable periauricular mass or external auricular canal polyps to nonspecific symptoms such as hearing loss, otalgia, or otorrhea. $^{3,4}$ Some patients may be completely asymptomatic. ${ }^{5}$ Because these diseases can easily mimic benign processes, the diagnoses of RMS and LCH are usually only considered after imaging reveals a skull base or temporal bone lesion. However, the imaging characteristics of these tumors are similar on both CT and MR imaging.

Much of the current literature focuses exclusively on either RMS or LCH. The objective of our study was to compare radiologic data from patients with both RMS and LCH to determine characteristics that may aid clinicians in differentiating these 2 pathologies. To our knowledge, this is the first study to compare the imaging characteristics of these lesions. We hypothesized that 
these lesions would have distinct characteristics on crosssectional imaging, which will aid in making the proper diagnosis. Understanding the likely pathology before an operation is undertaken will help with surgical planning. Previously held wisdom suggested that RMS lesions would arise from muscle and be most likely to affect the middle ear, given the presence of the tensor tympani and stapedius muscles in the middle ear. $\mathrm{LCH}$ lesions would be most likely to arise from the marrow space and therefore more commonly affect the mastoid. We designed a single blinded review of cross-sectional imaging studies in pathologically confirmed cases to determine the most common site in the temporal bone for each lesion and the type of tissue most likely affected.

\section{MATERIALS AND METHODS}

To find cases of LCH and RMS affecting the temporal bone, we used billing data to find all International Classification of Diseases, Ninth Revision codes involving benign or malignant lesions of the temporal bone, skull, and bone, and we cross-referenced these data with patients seen in our otolaryngology clinics. Charts were reviewed retrospectively. Each chart was reviewed to establish a tissue diagnosis of LCH or RMS and to ensure that cross-sectional images were available for review. Both MR imaging and CT studies were in our inclusion criteria, depending on the technique available for each patient. We also used a radiology data base of temporal bone lesions to search for additional cases of known RMS or LCH for which images were available for review.

Demographic information was collected along with the presenting signs and symptoms for each case. Sample images representative of the lesions or the entire scan were reviewed independently by both a neuroradiologist and neurotologist. The reviewers were blinded to the pathology of the lesion.

A subsite and tissue type classification was created to describe the location of the lesion within the temporal bone and the type of tissue from which it appeared to arise. The temporal bone subsites included the petrous apex, middle ear, mastoid, and retrosigmoid/posterior cranial fossa. The petrous apex and middle ear were defined as the anterior temporal bone, and the mastoid and retrosigmoid/posterior cranial fossa were defined as the posterior temporal bone. Each reviewer was asked to identify the involved sites within the temporal bone and classify the degree of involvement on a scale of 0 (no involvement), 1 (partial involvement), or 2 (complete involvement). Subsites that appeared affected but were not at the center of the lesion were graded as 0.5 .

Second, the type of tissue involved by the lesion was defined as being pneumatized bone, cortical bone, or the marrow space. The involvement of each type of tissue was again rated as 0 (no involvement), 1 (partial involvement), or 2 (complete involvement). Last, the reviewers were asked to rate otic capsule erosion as being present or absent.

Subsite, tissue type, and otic capsule erosion scores were compared with a $\chi^{2}$ test if possible, but the low number of cases also required the use of a Fisher exact test. Two-tailed $P$ values $<.05$ were statistically significant.

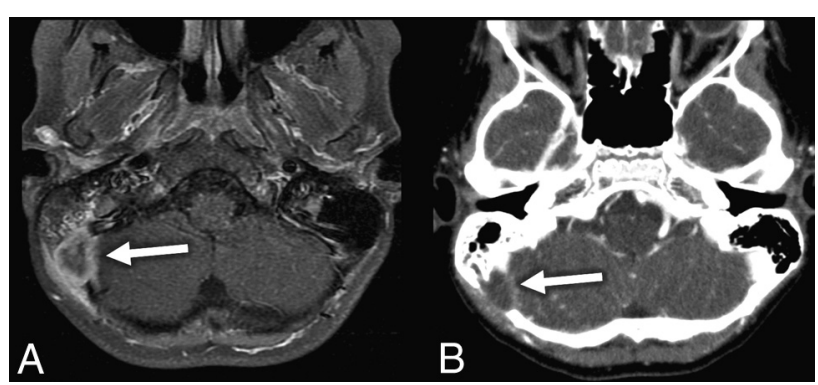

FIG 1. A, Axial T1 postcontrast fat-saturation MR image through the temporal bone demonstrates a peripherally enhancing posterior fossa or retrosigmoid LCH lesion (arrow). B, Axial standard algorithm postcontrast correlating CT scan demonstrates the same LCH lesion (arrow) with osseous destructive changes.

\section{RESULTS}

Our review of the records and the imaging data base identified 14 cases of LCH in 12 patients, with 2 patients having bilateral lesions, and 9 cases of RMS in 9 patients.

\section{LCH Cases}

Patients with LCH had an average age of 7.25 years and a male/ female ratio of 1.4:1. Most patients (11/12) presented with otologic symptoms, and 1 patient presented with only constitutional symptoms. The most common otologic symptom was swelling or a periauricular mass $(8 / 12)$. CT was the only imaging available in 8 of these cases, 1 had MR imaging only, and 3 additional cases had both MR imaging and CT performed.

The total for temporal bone subsite scores was 28 (14 cases with a possible total of 2 points each). The mastoid was the most commonly involved region with a score of 23/28 (82\%), followed by middle ear involvement with a score of $13.5 / 28$ (48\%). The other sites were involved less commonly: retrosigmoid/posterior fossa involvement was 6/28 (21\%) (Fig 1), and petrous apex involvement was $3 / 28(10 \%)$. Disease was confined entirely to the anterior region in $1 / 14$ ears and to the posterior region in $4 / 14$ ears, with the remaining $9 / 14$ cases having involvement in both the anterior and posterior temporal bone.

With respect to the tissue type at the center of the lesion, pneumatized bone was the most common area involved with a score of $24 / 28$ (85\%), the cortical bone was $11 / 28$ (39\%), and marrow space involvement was $3 / 28(10 \%)$. There was otic capsule erosion in 3 of 14 ears.

\section{RMS Cases}

Patients with RMS had an average age of 4.93 years with a male/ female ratio of 2.5:1. Most patients (8/9) presented with otologic symptoms, and only 1 patient presented with exclusively neurologic symptoms. The most common symptom was pain in $3 / 9$ patients. Two patients presented with a mass, 2 patients presented with infectious symptoms, and 2 patients presented with facial palsy. CT was the only imaging available in 3 of these patients, 2 patients had MR imaging only, and 4 additional patients had both MR imaging and CT performed.

The total for subsite scores for RMS was 18 ( 9 cases with a possible total of 2 points each). Middle ear involvement was most common with a subsite score of 9.5/18 (52\%). Mastoid involvement was $6 / 18(33 \%)$, petrous apex involvement was $6 / 18$ 


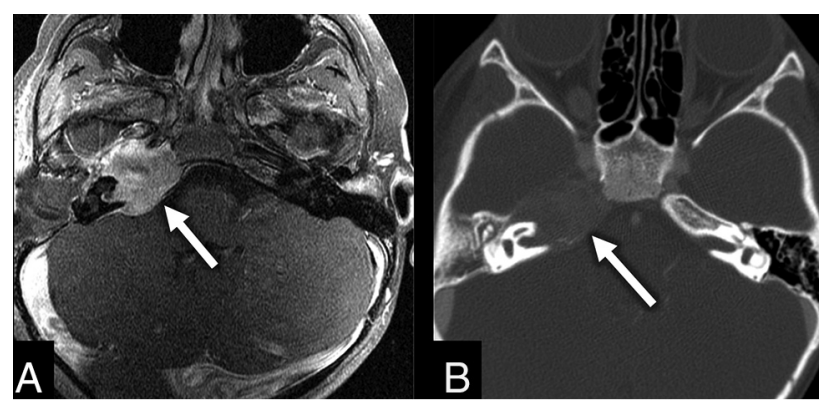

FIG 2. A, Axial fat-saturated postcontrast TI MR image through the temporal bone shows a heterogeneous avidly enhancing lesion within the right petrous apex (arrow) found to be an RMS. B, Axial postcontrast correlating CT image in a bone window shows the same lesion (arrow) with expansile changes to the right petrous apex.

Temporal bone subsite scores for LCH and RMS lesions and associated $P$ values

\begin{tabular}{lrrc}
\hline \multicolumn{1}{c}{ Subsite } & LCH & RMS & P Value \\
\hline Middle ear & $48 \%$ & $53 \%$ & .67 \\
Mastoid & $82 \%$ & $33 \%$ & .001 \\
Petrous apex & $11 \%$ & $33 \%$ & .12 \\
Retrosigmoid/posterior cranial fossa & $21 \%$ & $0 \%$ & .07 \\
\hline
\end{tabular}

(33\%) (Fig 2), and retrosigmoid/posterior fossa involvement was $0 / 18(0 \%)$. Disease was confined entirely to the anterior region of the temporal bone in 5/9 patients. The lesion involved only the posterior temporal bone in just $1 / 9$ patients. The remaining 3 patients had involvement of the anterior and posterior temporal bone.

The most commonly involved area was in pneumatized bone in $11 / 18(61 \%)$ scores, followed by marrow space involvement with a score of $6 / 18(33 \%)$ and, least commonly, cortical bone with $2 / 18$ (11\%). There was otic capsule erosion in 2 of 9 ears.

Using a $\chi^{2}$ analysis, we noted a statistically significant difference with respect to the involvement of the mastoid in $\mathrm{LCH}, 82 \%$, and in RMS, 33\%, $(P=.001)$. The involvement of the remaining subsites was not significantly different between RMS and LCH. Please see the Table for the remaining subsite scores and associated $P$ values. With the Fisher exact test, our data showed that confinement to the anterior region exclusively was significantly more likely in patients with RMS, 56\%, versus $7.1 \%$ with $\mathrm{LCH}$ $(P=.018)$.

Interrater reliability as measured by a weighted $\kappa$ was moderate for the middle ear location, good for the mastoid and retrosigmoid/posterior cranial fossa locations, and very good for the petrous apex location $(P<.05$ for all comparisons).

Additionally, we found a significant difference in the rate of the cortical bone involvement, with LCH being more common in $39 \%$ of cases compared with $11 \%$ in RMS $(P=.05)$. For the type of bone involved, interrater reliability was very good for pneumatized bone and marrow space $(P<.05)$, but the reliability for cortical bone involvement did not reach statistical significance $(P=.09)$. Thus, the involvement of cortical bone was not considered statistically significant. The data did not indicate significant differences in the type of bone involved, erosion of the otic capsule, or the presence of bilateral lesions.

\section{DISCUSSION}

Patients with RMS and LCH of the temporal bone frequently initially present with nonspecific symptoms. Both of these lesions occur most commonly in the pediatric population, which also has a high rate of benign ear disease, making the early diagnosis of a neoplastic process more difficult, yet critical in these complex cases.

\section{Langerhans Cell Histiocytosis}

LCH represents a spectrum of diseases from isolated lesions (eosinophilic granulomas) to multifocal disease (Hand-SchullerChristian disease) and last to widespread disease with multiorgan involvement (Letterer-Siwe disease). The reported incidence of $\mathrm{LCH}$ involvement of the temporal bone varies widely from $13 \%$ to $40 \%$ of cases. Lesions tend to involve the marrow space and can arise from the diploic bone of the cranial vault. ${ }^{6}$ Lesions should be definitively diagnosed by histology demonstrating a proliferation of Langerhans cells and staining positive for S100 and CD1a on immunohistochemistry. ${ }^{4}$

Presentation of temporal bone involvement of $\mathrm{LCH}$ is most often with nonspecific otologic signs and symptoms, which could be mistaken for chronic otitis media or other infectious processes. According to published series, the most common presentations are a periauricular mass or otitis media. There is a slight male predominance of $\mathrm{LCH}$ cases of the temporal bone in the literature. $^{4,7}$

Consistent with the findings reported in this study, the series of Fernández-Latorre et $\mathrm{al}^{8}$ of LCH cases demonstrated 14 total cases with 2 patients having bilateral lesions. In their series, the site-specific frequency was 12 cases involving the mastoid, 9 involving the middle ear, and 2 cases involving the petrous apex. Similar to our series, several lesions involved multiple temporal bone subsites. Additionally, the series had 2 cases of otic capsule erosion. In another review of head and neck LCH, there was temporal bone involvement in the mastoid in 4 of 5 cases and in the petrous bone in 1 of 5 cases. ${ }^{7}$ Another case series noted "rare" otic capsule involvement. ${ }^{4}$ Treatment for $\mathrm{LCH}$ varies on the basis of the severity of the disease and includes surgical excision, chemotherapy, radiation, and intratumoral steroids. Given the relatively benign nature of isolated LCH lesions, some authors have argued that the therapy should not be worse than the disease. ${ }^{9}$

\section{Rhabdomyosarcoma}

RMS is a rare disease of the pediatric temporal bone. There are 4 distinct pathologic types of RMS: embryonal (most common in head and neck ${ }^{10}$ ), alveolar, pleomorphic, and botryoid. Tumors can arise from striated muscle or from undifferentiated mesenchymal cells. Desmin and muscle-specific actin stains are helpful in identifying RMS lesions. In 2 series, presentation was at approximately 4 years of age. ${ }^{10}$ Notably, there are no reported cases of bilateral temporal bone RMS, in contrast to reports of bilateral temporal bone $\mathrm{LCH}$. There is a slight male predominance in RMS patients. $^{10}$

The presentation of RMS is most commonly mistaken for benign ear disease, resulting in a reported delay in diagnosis ranging from 4 to 78 weeks. ${ }^{10}$ Chronic otitis media, polyps of the external auricular canal, and retroauricular masses are all common pre- 
sentations. ${ }^{3,10}$ Other series showed a much higher prevalence of neurologic signs, including a series in which 9 of 14 patients presented with facial nerve palsies. ${ }^{10}$

Among patients with RMS lesions of the temporal bone, 50\% had lesions that were localized to a specific temporal bone subsite, and the remaining $50 \%$ had extratemporal extension $(n=6){ }^{3}$ The staging system for RMS classifies a T1 lesion as being limited to 1 subsite of the temporal bone (mastoid, middle ear, or petrous apex). ${ }^{11}$ The middle ear is considered an unfavorable subsite for RMS staging. ${ }^{12}$ RMS cases affecting the bilateral temporal bones have not been reported, to our knowledge. Cases of RMS in the temporal bone have poorer prognosis than RMS of the orbit and the extratemporal head and neck. ${ }^{3}$

It has been reported that the most common site affected by RMS is the mastoid (57\%), followed by the middle ear $(43 \%)$, petrous apex (35\%), and posterior fossa (14\%), while $50 \%$ of cases affected multiple temporal bone subsites. ${ }^{10} \mathrm{~A}$ series published by Sbeity et $\mathrm{al}^{3}$ demonstrated RMS involvement of the middle ear in $50 \%$ of lesions, the mastoid in $50 \%$, and the petrous apex in $17 \%$. In a case reported by Viswanatha, ${ }^{13}$ the RMS lesion affected both the middle ear and mastoid. The role of surgery in treating RMS cases is variable, depending on the series. If the lesion can be completely excised, the prognosis improves regardless of the subsite. Most patients will be treated with chemotherapy, and 5-year survival rates vary from $66 \%{ }^{3}$ to $90 \%$ in patients with resectable tumors. ${ }^{14}$

\section{Imaging in LCH and RMS}

In patients who ultimately are diagnosed with either RMS or $\mathrm{LCH}$, imaging is often performed when initial treatments for otitis media and cholesteatoma fail. Each lesion has some imaging features that overlap with the imaging features of cholesteatoma and middle ear effusion. There are findings that help to differentiate these lesions on imaging.

On CT, RMS usually demonstrates a homogeneous lesion with mild contrast enhancement. On MR imaging, it has intermediate signal intensity on T1 and T2 sequences with significant enhancement with contrast. From the series of Sbeity et al, ${ }^{3} 1$ case was localized to the mastoid; 1 , to the middle ear; and 1, to the petrous bone. ${ }^{3}$ Because RMS does not arise from the bone, bony erosion is considered a poor prognostic sign and indicates a T3 lesion. ${ }^{11}$

In the 1984 review of Coutte et al, ${ }^{15}$ CT findings could not distinguish cholesteatoma and LCH in children who presented with acute otitis media. Most series reported LCH lesions as being lytic or destructive. ${ }^{16-18}$ There was frequently an associated softtissue attenuation or a soft-tissue mass, and the otic capsule was usually spared. ${ }^{6}$ Lesions can have beveled edges because the LCH lesion seems to affect the inner and outer tables asymmetrically. Button sequestrum is a finding associated with lytic changes surrounding an area of apparently normal bone, ${ }^{19}$ though this process may not be appreciable in the 3D contours of the temporal bone.

MR imaging sequences show nonspecific changes consistent with inflammation and bone marrow involvement. MR imaging can better demonstrate intracranial extension and associated softtissue involvement, as well as distinguish these lesions from cholesteatoma. The MR imaging features of a cholesteatoma will in- clude isointensity on $\mathrm{T} 1$, with slight hyperintensity compared with brain on T2, and no enhancement of the lesion. The lack of enhancement along with restricted diffusion in cholesteatoma cases is the critical imaging feature to differentiate $\mathrm{LCH}$ from cholesteatoma and demonstrates the improved soft-tissue evaluation of MR imaging over CT.

In our case series, only 2 of our 21 patients had a suspicion of an RMS or LCH before cross-sectional imaging. Cross-sectional imaging of these patients can suggest the underlying pathology of the lesion when the subsites of involvement are carefully evaluated. In all cases, a tissue diagnosis was made histologically. To our knowledge, this is the first series to compare imaging findings between these 2 uncommon pediatric temporal bone lesions. Lesions involving the mastoid subsite are significantly more likely to be LCH than RMS. Lesions involving the anterior portion of the temporal bone, including the petrous apex and middle ear, are more likely to be RMS.

The scope of this study was limited by its retrospective nature and the heterogeneity of imaging modalities available. Consequently, analysis of certain other features such as the presence of hemorrhage, necrosis, stalk thickening, or extratemporal involvement or comparison of ADC values could not be performed. Evaluating these other imaging characteristics could be helpful in the differentiation of these lesions for future research.

\section{CONCLUSIONS}

Rhabdomyosarcoma and Langerhans cell histiocytosis are both rare lesions of the pediatric temporal bone. Diagnosis usually occurs when patients fail standard treatment of suspected infectious causes or develop masses of the ear or temporal bone and imaging is undertaken. The imaging characteristics for these lesions are similar; however, the location of the lesion can be helpful in making a correct diagnosis. Our results indicate that the subsite of temporal bone involvement and the anterior or posterior nature of the lesion on imaging suggest the pathology of the lesion and allow better treatment planning before tissue diagnosis. LCH more frequently involves the mastoid, and RMS is typically confined to only the anterior temporal bone compared with LCH.

\section{REFERENCES}

1. Zhang X, Ma K, Wang J, et al. A prospective evaluation for the combined helical tomotherapy and chemotherapy in pediatric patients with unresectable rhabdomyosarcoma of the temporal bone. Cell Biochem Biophys 2014;70:103-08 CrossRef Medline

2. Martini A, Aimoni C, Trevisani C, et al. Langerhans' cell histiocytosis: report of a case with temporal localization. Int J Pediatr Otorhinolaryngol 2000;55:51-56 CrossRef Medline

3. Sbeity S, Abella A, Arcand P, et al. Temporal bone rhabdomyosarcoma in children. Int J Pediatr Otorhinolaryngol 2007;71:807-14 CrossRef Medline

4. Saliba I, Sidani K, El Fata F, et al. Langerhans' cell histiocytosis of the temporal bone in children. Int J Pediatr Otorhinolaryngol 2008;72: 775-86 CrossRef Medline

5. Yildirim-Baylan M, Cureoglu S, Paparella MM. Langerhans' cell histiocytosis of the temporal bone. Otol Neurotol 2012;33:e15-16 CrossRef Medline

6. Azouz EM, Saigal G, Rodriguez MM, et al. Langerhans' cell histiocytosis: pathology, imaging and treatment of skeletal involvement. Pediatr Radiol 2005;35:103-15 CrossRef Medline 
7. Anonsen CK, Donaldson SS. Langerhans' cell histiocytosis of the head and neck. Layngoscope 1987;97:537-42 Medline

8. Fernández-Latorre F, Menor-Serrano F, Alonso-Charterina S, et al. Langerhans' cell histiocytosis of the temporal bone in pediatric patients: imaging and follow-up. AJR Am J Roentgenol 2000;174: 217-21 CrossRef Medline

9. Boston M, Derkay CS. Langerhans' cell histiocytosis of the temporal bone and skull base. Am J Otolaryngol 2002;23:246-48 CrossRef Medline

10. Durve DV, Kanegaonkar RG, Albert D, et al. Paediatric rhabdomyosarcoma of the ear and temporal bone. Clin Otolaryngol Allied Sci 2004;29:32-37 CrossRef Medline

11. Donaldson CS, Castro JR, Wilbur JR, et al. Rhabdomyosarcoma of the head and neck in children: combination treatment by surgery, irradiation, and chemotherapy. Cancer 1973;31:26-36 Medline

12. Lawrence W Jr, Anderson JR, Gehan EA, et al. Pretreatment TNM staging of childhood rhabdomyosarcoma: a report of the Intergroup Rhabdomyosarcoma Study Group. Cancer 1997;80:1165-70 Medline
13. Viswanatha B. Embryonal rhabdomyosarcoma of the temporal bone. Ear Nose Throat J 2007;86:218-22 Medline

14. Baker KS, Anderson JR, Link MP, et al. Benefit of intensified therapy for patients with local or regional embryonal rhabdomyosarcoma: results from the Intergroup Rhabdomyosarcoma Study IV. J Clin Oncol 2000;18:2427-34 Medline

15. Coutté A, Brahe Pedersen C, Bartholdy N, et al. Histiocytosis X; recurrent otitis media as a presenting symptoms in children with special reference to cholesteatoma. Clin Otolaryngol Allied Sci 1984;9: 111-14 CrossRef Medline

16. Hadjigeorgi C, Parpounas C, Zarmakoupis, et al. Eosinophilic granuloma of the temporal bone: radiological approach in the pediatric patient. Pediatr Radiol 1990;20:546-49 CrossRef Medline

17. Neilan RE, Kutz JW Jr. Langerhans cell histiocytosis of the temporal bone. Otol Neurotol 2012;33:e31-32 CrossRef Medline

18. Nelson BL. Langerhans cell histiocytosis of the temporal bone. Head Neck Pathol 2008;2:97-98 CrossRef Medline

19. Stull MA, Kransdorf MJ, Devaney KO. Langerhans cell histiocytosis of bone. Radiographics 1992;12:801-23 CrossRef Medline 